\title{
Preexisting oncogenic events impact trastuzumab sensitivity in ERBB2-amplified gastroesophageal adenocarcinoma
}

\author{
Jihun Kim, ${ }^{1,2,3}$ Cameron Fox, ${ }^{1}$ Shouyong Peng, ${ }^{1,2}$ Mark Pusung, ${ }^{1}$ Eirini Pectasides, ${ }^{1,4}$ Eric Matthee, ${ }^{1,5}$ Yong Sang Hong, ${ }^{1,6}$ In-Gu Do, ${ }^{7}$ \\ Jiryeon Jang, ${ }^{8}$ Aaron R. Thorner, ${ }^{9}$ Paul Van Hummelen, ${ }^{1,9}$ Anil K. Rustgi, ${ }^{10}$ Kwok-Kin Wong, ${ }^{1,11}$ Zhongren Zhou, ${ }^{12}$ Ping Tang, ${ }^{13}$ \\ Kyoung-Mee Kim, ${ }^{7}$ Jeeyun Lee, ${ }^{8}$ and Adam J. Bass ${ }^{1,2,11,14}$

\begin{abstract}
'Department of Medical Oncology, Dana-Farber Cancer Institute, Boston, Massachusetts, USA. ${ }^{2}$ Cancer Program, The Broad Institute of MIT and Harvard, Cambridge, Massachusetts, USA. ${ }^{3}$ Department of Pathology, University of Ulsan College of Medicine, Asan Medical Center, Seoul, Republic of Korea. ${ }^{4}$ Department of Medicine, Beth Israel Deaconess Medical Center, Boston, Massachusetts, USA. ${ }^{5}$ Radboud University Nijmegen Medical Centre, Nijmegen, The Netherlands. ${ }^{6}$ Department of Internal Medicine, University of Ulsan School of Medicine, Asan Medical Center, Seoul, Republic of Korea. ${ }^{7}$ Department of Pathology and ${ }^{8}$ Department of Internal Medicine, Samsung Medical Center, Sungkyunkwan University School of Medicine, Seoul, Republic of Korea. ${ }^{9}$ The Center for Cancer Cenome Discovery, Dana-Farber Cancer Institute, Boston, Massachusetts, USA. ${ }^{10}$ Department of Medicine, University of Pennsylvania School of Medicine, Philadelphia, Pennsylvania, USA. "Department of Medicine, Harvard Medical School, Boston, Massachusetts, USA. ${ }^{12}$ Department of Surgery and ${ }^{13}$ Department of Pathology and Laboratory Medicine, University of Rochester, Rochester, New York, USA. ${ }^{14}$ Department of Medicine, Brigham and Women's Hospital, Boston, Massachusetts, USA.
\end{abstract}

Patients with gastric and esophageal (GE) adenocarcinoma tumors in which the oncogene ERBB2 has been amplified are routinely treated with a combination of cytotoxic chemotherapy and the ERBB2-directed antibody trastuzumab; however, the addition of trastuzumab, even when tested in a selected biomarker-positive patient population, provides only modest survival gains. To investigate the potential reasons for the modest impact of ERBB2-directed therapies, we explored the hypothesis that secondary molecular features of ERBB2-amplified CE adenocarcinomas attenuate the impact of ERBB2 blockade. We analyzed genomic profiles of ERBB2-amplified CE adenocarcinomas and determined that the majority of ERBB2-amplified tumors harbor secondary oncogenic alterations that have the potential to be therapeutically targeted. These secondary events spanned genes involved in cell-cycle regulation as well as phosphatidylinositol-3 kinase and receptor tyrosine kinase signaling. Using ERBB2-amplified cell lines, we demonstrated that secondary oncogenic events could confer resistance to ERBB2-directed therapies. Moreover, this resistance could be overcome by targeting the secondary oncogene in conjunction with ERBB2-directed therapy. EGFR is commonly coamplified with ERBB2, and in the setting of ERBB2 amplification, higher ECFR expression appears to mark tumors with greater sensitivity to dual EGFR/ERBB2 kinase inhibitors. These data suggest that combination inhibitor strategies, guided by secondary events in ERBB2-amplified GE adenocarcinomas, should be evaluated in clinical trials.

\section{Introduction}

Gastric adenocarcinoma is the fourth most common cancer and the third leading cause of cancer deaths worldwide, and the incidence of esophageal adenocarcinoma is increasing dramatically in many Western countries $(1,2)$. Since traditional cytotoxic chemotherapy has not substantially improved survival rates, more hope has been placed in the potential of newer targeted therapies that will provide improvements in outcomes similar to those they have achieved in other cancers (3-7). The only targeted therapeutic agent in routine use for first-line treatment of gastric and esophageal (GE) adenocarcinoma is the anti-ERBB2 monoclonal antibody trastuzumab, which is used for the $~ 15 \%$ of patients with ERBB2 overexpression and/or amplification. The addition of trastuzumab to empiric chemotherapy, however, led to an improvement in survival of only 2.7 months for ERBB2-positive patients

Authorship note: Jihun Kim and Cameron Fox contributed equally to this work. Conflict of interest: The authors have declared that no conflict of interest exists. Submitted: January 14, 2014; Accepted: October 14, 2014.

Reference information: J Clin Invest. 2014;124(12):5145-5158. doi:10.1172/JCI75200. and increased clinical response rates from $35 \%$ to only $47 \%$ (8). Furthermore, a parallel study evaluating the addition of the ERBB2/EGFR dual inhibitor lapatinib to chemotherapy revealed a similar increase in response rates but no significant improvement in survival (9). These data suggest that de novo or intrinsic resistance of ERBB2-amplified GE adenocarcinomas to ERBB2 inhibition must be overcome to improve clinical outcomes.

Most studies investigating resistance to trastuzumab have emanated from the breast cancer field. Acquired resistance to ERBB2 therapy in breast cancer can follow the activation of alternative signaling pathways involving phosphatidylinositol 3-kinase (PI3K) (10-14), SRC, EGFR, and IGF1R and the cell-cycle regulator cyclin E1 (15-18). Further studies have investigated the impact on trastuzumab sensitivity of other ERBB members in breast cancer. For example, trastuzumab reportedly had the greatest benefit for ERBB2-amplified breast cancer driven by ERBB2:ERBB2 homodimers (19). Moreover, ERBB3:ERBB2 dimerization has been reported in many $E R B B 2$-amplified breast cancers (20), leading to investigation of the addition of ERBB3 inhibitors to ERBB2directed therapy (21-24). 
By contrast, substantially less effort has been devoted to investigating the means of intrinsic or acquired resistance to trastuzumab and other ERBB2-targeting agents in GE adenocarcinomas. Based on cell line models, activation of the cAMP-regulated phosphoprotein t-DARPP (25) and the kinase SRC (16) has been shown to mediate acquired resistance to trastuzumab. However, it is not known which pathways are responsible for the de novo or intrinsic resistance that many ERBB2-positive patients demonstrate with ERBB2-directed therapy.

In earlier studies, we demonstrated that the genomes of primary, previously untreated GE adenocarcinoma have a rich spectrum of somatic copy-number alterations (26) and point mutations (27). Based on these observations, we hypothesized that systematic analysis of the preexisting oncogenic mutations and copy-number alterations that often co-occur with ERBB2 amplification in GE adenocarcinomas would reveal possible etiologies of intrinsic or de novo resistance of many ERBB2-positive tumors to ERBB2-directed therapy. These genomic studies reveal that, even prior to therapy, ERBB2 amplification in GE adenocarcinoma often occurs in the context of coexisting, de novo alterations involving the PI3K pathway, other receptor tyrosine kinases (RTKs), or cell-cycle mediators. We followed these genomic observations by demonstrating in in vitro models that the presence of these co-occurring alterations can lead to intrinsic resistance to ERBB2directed therapy and that resistance could be attenuated through the combination of an ERBB2 inhibitor and an inhibitor of the secondary alteration. Through these studies, we observed that EGFR amplification co-occurs in a subset of untreated ERBB2-positive GE adenocarcinomas and identified that a subset of tumors harbors elevated EGFR expression, even in the absence of an EGFR genomic alteration. We demonstrated that in the setting of higher EGFR expression, there was marked dimerization of ERBB2 with EGFR, thus identifying a subset of tumors in which EGFR/ERBB2 dual inhibitors may have greater efficacy than the ERBB2-directed antibody therapy currently used in patients.

These data suggest that secondary features contribute to the intrinsic resistance of many ERBB2-positive GE adenocarcinomas to current therapies. By identifying key preexisting secondary genomic and molecular features of these tumors, we may be able to develop rational, biomarker-guided combination approaches to improve therapies for these cancers.

\section{Results}

Additional oncogenic events frequently co-occur with ERBB2 amplification in GE adenocarcinomas. We first sought to identify the spectrum of baseline genomic alterations present within a population of ERBB2-amplified GE adenocarcinomas that had not been subjected to prior therapies. Since our previous studies demonstrated that the profiles of both chromosome arm-level and focal somatic copy-number aberrations of GE adenocarcinoma were more similar to each other than to any other cancer type (28), we decided to pool GE cancer data. We collected publicly available data from untreated, fresh-frozen primary GE adenocarcinomas subjected to copy-number analysis using SNP6 Affymetrix SNP arrays. Between samples from our earlier publication (26), other published studies (29), and The Cancer Genome Atlas (TCGA) (30), we compiled data on $743 \mathrm{GE}$ adenocarcinomas and found
62 ERBB2-amplified cases. As in prior studies of SNP array-based copy numbers, amplification was defined as the estimated copy number greater than 3.7. Since the presence of non-neoplastic cells in the tumor samples attenuate the copy-number intensity, these values will systematically underestimate the actual degree of amplification in the tumor. In the genomes of these 62 tumors, we analyzed the spectrum of copy-number alterations using the GISTIC algorithm to locate genomic loci that are subject to statistically recurrent focal amplifications. With this unbiased, genomewide analysis, we identified significant foci of amplifications that localize to CCNE1, CDK6, EGFR, MET, and MYC (Figure 1A and Supplemental Table 1; supplemental material available online with this article; doi:10.1172/JCI75200DS1) in addition to the expected $E R B B 2$ peak on $17 \mathrm{q} 12$ and secondary peaks around $E R B B 2$. Among these, the most statistically significant secondary alteration was the focal amplification at chromosome 19q12, which was localized by the GISTIC algorithm to a peak containing a single gene, CCNE1, encoding the cell-cycle regulator cyclin E1 (Supplemental Figure 1). The clear focality of the amplifications at the CCNE1 locus, coupled with the established role of CCNE1 amplifications in ovarian cancer (31), suggest that CCNE1 amplifications are also critical events in $\mathrm{ERBB}^{+} \mathrm{GE}$ adenocarcinomas.

To assist the interpretation of these findings regarding candidate oncogenes coamplified in untreated ERBB2-amplified GE adenocarcinomas, we performed a similar analysis of 103 (11.2\%) $E R B B 2$-amplified breast cancers (culled from 916 fresh-frozen untreated tumors profiled through TCGA; ref. 32). Within ERBB2amplified breast tumors, the CCND1 locus was the only significantly coamplified oncogene, thus suggesting that coamplification of other secondary oncogenes is more prominent in ERBB2 ${ }^{+}$ GE adenocarcinomas (Supplemental Figure 2 and Supplemental Table 2). When we examined individual tumors, several GE adenocarcinomas showed striking amplifications of CCNE1, CDK6, and $M E T$ loci, whereas breast cancers showed frequent CCND1 amplifications (Figure 1B). EGFR amplifications were present in both tumors types but were more common in the GE adenocarcinomas $(7.8 \%$ vs. $1.6 \%)$. We compared the frequency of these amplifications in the 62 ERBB2-amplified GE adenocarcinomas with those cases without $E R B B 2$ amplification and found that CCNE1, CDK6, EGFR, and MET amplifications were present at a statistically greater frequency in the ERRB2 ${ }^{+}$cohort when compared with ERBB2- samples (Supplemental Table 3).

We next sought to determine whether potentially oncogenic point mutations are also present in these tumors. We queried the 42 ERBB2-amplified GE adenocarcinomas with available whole-exome sequencing data and found mutations putatively activating the PI3K pathway in 8 of $42(19.0 \%)$ cases. These mutations impacted PIK3CA, PIK3R1, and PTEN (Figure 2B and Supplemental Table 4). The PIK3CA mutations at codons 542 and 545 are well-known canonical activating mutations. In addition, 2 of the 3 PIK3R1 mutations were nonsense alterations predicted to truncate the protein. As other truncating PIK3R1 events observed in endometrial cancer have been demonstrated to activate AKT through the destabilization of PTEN (33), the presence of these mutations in ERBB2 ${ }^{+} \mathrm{GE}$ adenocarcinoma can also reasonably be assumed to activate the PI3K pathway. Several other mutations involving the PI3K pathway, PIK3CA codon 600, PTEN codon 
A

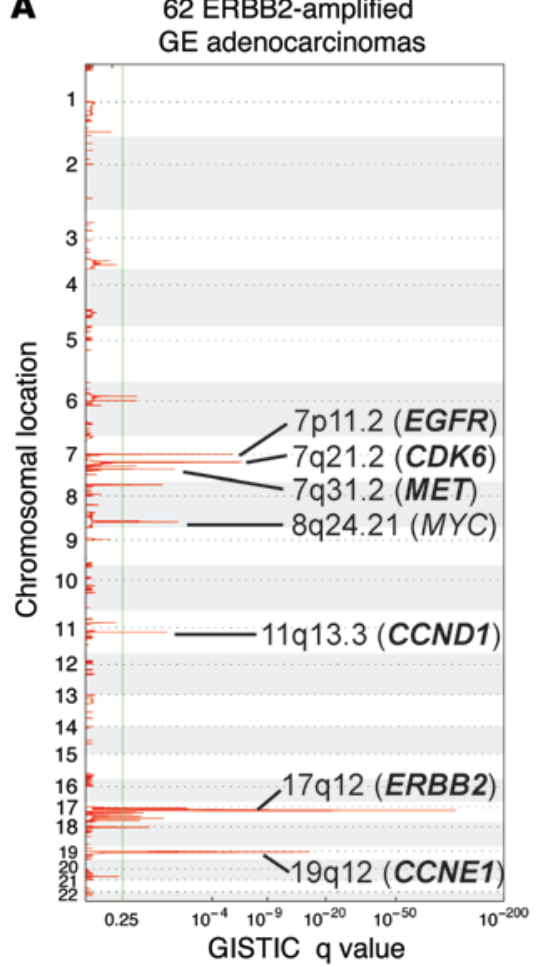

B
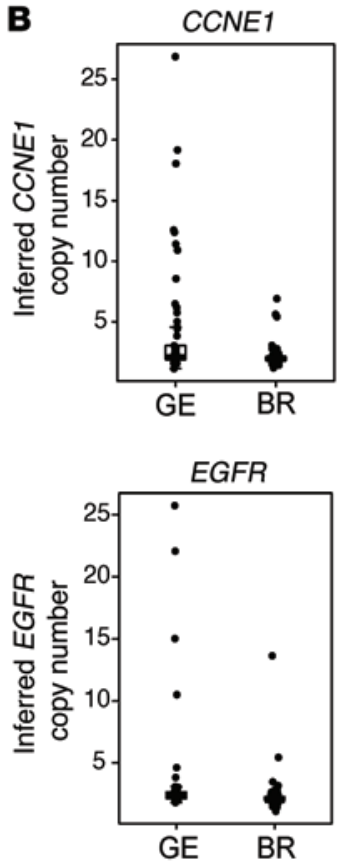
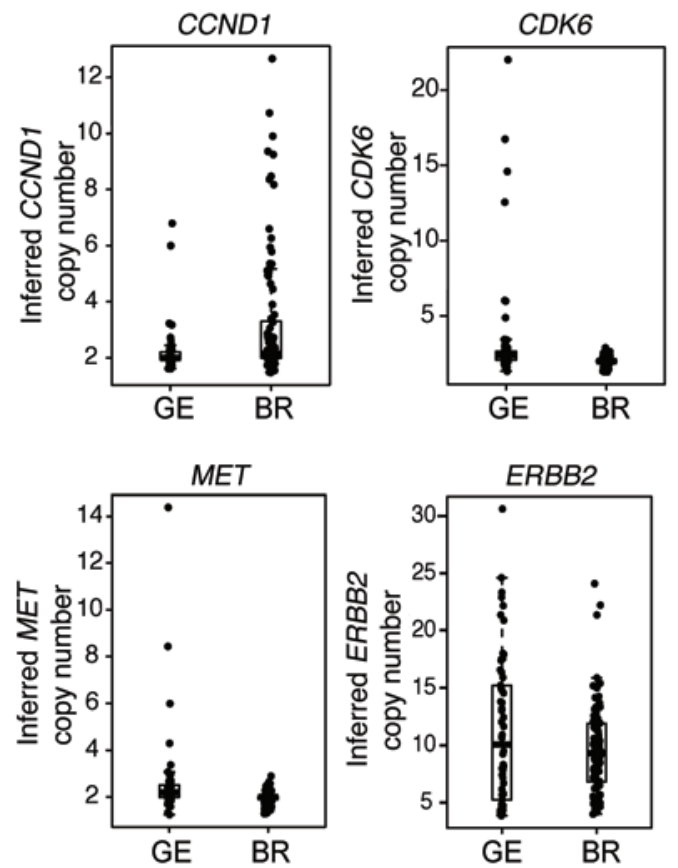

Figure 1. Co-occurring oncogenic amplifications in ERBB2-amplified GE adenocarcinoma samples. (A) GISTIC plot of 62 ERBB2-amplified GE cancers ( $x$ axis: corrected q value; $y$ axis: chromosomal coordinates) exhibits several significant focal amplifications involving important oncogenes (annotated in the right column). (B) Estimated copy numbers of the significantly coamplified oncogenes are depicted for ERBB2-amplified $\mathrm{CE}$ ( $n=62$ ) and breast $(n=103)$ cancer samples. Each dot represents an individual case, and the horizontal bar indicates the mean value. BR, breast.

28, and AKT3 codon 287, were of unclear pathogenic significance (Supplemental Table 4). We also found 6 mutations in RTKs such as ERBB3 $(n=3), \operatorname{MET}(n=2)$, and FGFR2 $(n=1)$. Although the amino acid positions of 3 of these specific mutations (ERBB3 M60K, MET T602fs, and FGFR2 P413R; summarized in Supplemental Table 4) had been reported in other cancer samples, the functional importance of these mutations is not known.

Integrated analysis of amplified and mutant events in these cases revealed that 23 of the 42 samples (54.8\%) had a co-occurring genomic event of potential functional significance (Figure 2A) when we included all amplifications of established oncogenes and point mutations of established functional relevance. Grouped by the shared pathways, we observed amplifications of the cell-cyclerelated genes in 17 samples (40.5\%), activating PI3K pathway alterations in 6 samples (11.9\%), and amplifications of other RTKs in 6 samples (14.3\%). Many of these were mutually exclusive, but cell-cycle gene alterations co-occurred with RTK alterations in 4 samples and with PI3K pathway alterations in 3 samples. Separating the profiles of tumors of gastric origin from those of esophageal origin revealed similar patterns of alterations in the 2 groups (Supplemental Figure 3). By comparison, in ERBB2 ${ }^{+}$breast cancer, we identified 100 cases from TCGA with matched copy-number and exome data. In this group of tumors, 42 (42\%) harbored similar secondary genomic alterations of potential functional significance (Supplemental Figure 4).

One limitation of our analysis is that we defined ERBB2 status based on copy number alone, whereas clinical diagnostics, which guide trastuzumab, also incorporate evidence of ERBB2 overexpression. To determine whether secondary oncogenes are present in GE tumors with clear overexpression of $E R B B 2$, we first queried matched SNP array and expression data from TCGA gastric cancer study and found that tumors with 10 or more copies of ERBB2 showed marked mRNA overexpression (Supplemental Figure 5). Across our total of 743 cases, we identified 31 tumors with 10 or more copies of ERBB2. Among these, there were 10 cases (32\%) with an amplified cell-cycle mediator (CCNE1, CDK6, or CCND1) and 4 cases with another amplified RTK (EGFR, ERBB3, or MET) (Supplemental Figure 6). We had 17 cases with 10 or more copies of $E R B B 2$ with matched exome data, of which 1 case harbored a PIK3CA mutation. This subanalysis indicates that secondarily activated oncogenes are frequently present in cases with highlevel $E R B B 2$ amplifications.

Concurrent PIK3CA mutation confers in vitro resistance to ERBB2 inhibitors that can be reversed through the use of therapies targeting the PI3K pathway. As a foundation to evaluate ERBB2 dependency in GE adenocarcinomas, we evaluated the sensitivity of a panel of ERBB2-amplified GE adenocarcinoma cell lines (Supplemental Table 5) to the EGFR/ERBB2 inhibitor lapatinib and found a range of sensitivities. Among these cell lines, NCI-N87 and OE19 had greater in vitro sensitivity to the ERBB2 inhibitor. By contrast, ESO26, OE33, and MKN7 cell lines had substantially less growth inhibition. We therefore asked whether other genomic alterations could impact the response of these models to lapatinib. We found that ESO26, which harbors an endogenous 


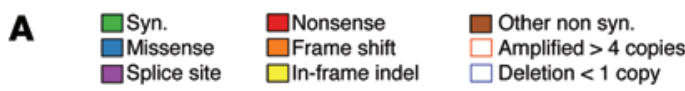

42 ERBB2-amplified GE adenocarcinoma cases with matched whole-exome sequencing and copy-number data
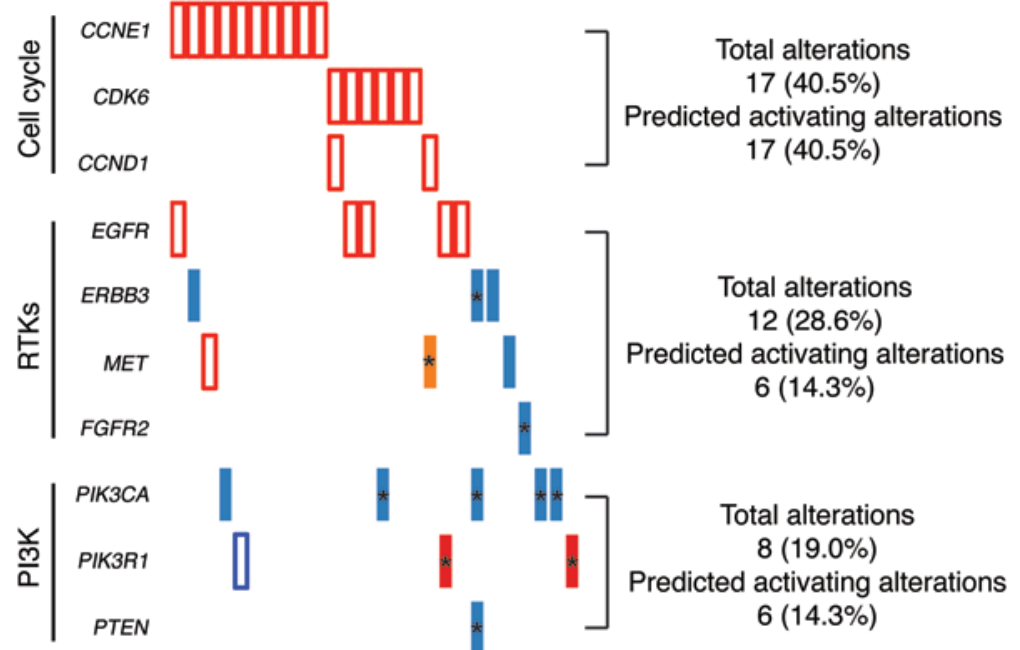

Cases (n= 42) :

Total alterations 26 (61.9\%)

Predicted activating alterations 23 (54.8\%)

B

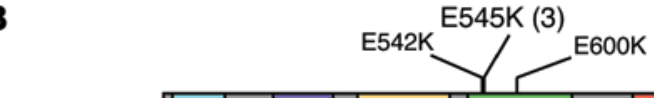

PIKЗCA $5 / 42(11.9 \%)$
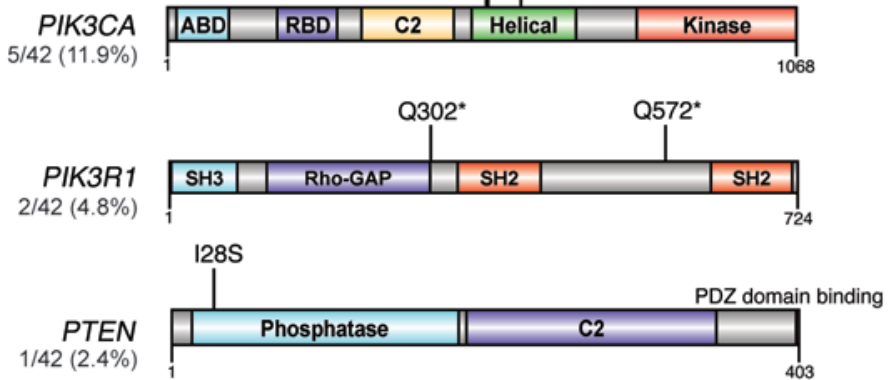

Figure 2. Potentially targetable oncogenic alterations in 42 ERBB2-amplified GE adenocarcinomas with available whole-exome sequencing and copy-number data. (A) Integrated view of co-occurring somatic oncogenic alterations in 42 CE adenocarcinomas. Potentially targetable oncogenes are listed by sample. Each column denotes an individual tumor, and each row displays a gene. Mutations are color coded by the type of mutation, and amplifications are depicted as red outlines. Mutations that occurred on the COSMIC-annotated amino acid are marked with asterisks (the amino acid impacted by the mutation was found in the COSMIC database). Predicted activating events include amplifications of cell-cycle and kinase genes and mutations at "hotspot" sites of PIK3CA and PIK3R1. (B) Schematic of co-occurring PI3K pathway gene mutations. All depicted mutations hit biologically significant domains such as helical, phosphatase, and kinase domains. Conserved domain mapping is from UniProt. ABD, adaptor-binding domain; COSMIC, Catalogue of Somatic Mutations in Cancer; RBD, RAS-binding domain; SH, SRC homology.
PIK3CA mutation at codon $546(\mathrm{Q} 546 \mathrm{H})$, immediately adjacent to the canonically mutated codon 545 , was less sensitive to lapatinib than were NCI-N87 or OE19, ERBB2-amplified cell lines lacking co-occurring genomic events that would be predicted to mitigate ERBB2 inhibitor effects (Figure 3A). ESO26 was furthermore resistant in vitro to trastuzumab (Supplemental Figure 7A). In addition, OE33 cells, harboring a co-occurring MET amplification and MKN7 cells, with a co-occurring CCNE1 amplification and PTEN deletion, were resistant to lapatinib and trastuzumab (Figure 3A and Supplemental Figure 7, B and C)

We next evaluated the ability of inhibition of the PI3K pathway to augment lapatinib sensitivity. We first compared the biochemical effects of lapatinib in the ESO26 cell line with those of the highly lapatinib-sensitive NCI-N87 line. Although ERBB2 phosphorylation was completely blocked by lapatinib treatment in both cell lines, the phosphorylation of AKT, a downstream effector of PIK3CA, persisted in ESO26 cells, but was inhibited in the NCI-N87 cells (Figure 3, B and C). We evaluated the ability of a small-molecule inhibitor of the p110 subunit of PI3K - GDC0941 - to augment the response of ESO26 to lapatinib and found growth inhibition (Figure 3E) and successful blockade of the persistent phosphorylation of AKT in ESO26 cells (Figure 3F). More formal synergy testing of GDC-0941 and lapatinib in the ESO26 cell line revealed a synergistic effect $(\mathrm{CI}=0.3866)$ (Supplemental Table 6). Additionally, we observed a similarly enhanced sensitivity when GDC-0941 was added to trastuzumab in the ESO26 cells (Supplemental Figure 7A). Furthermore, the MKN7 model, with ERRB2 amplification in addition to CCNE1 amplification and PTEN deletion, had a modest synergistic effect from the addition of PI3K inhibition to lapatinib, although not as substantial as that observed in the ESO26 model. To determine whether the ability of the PI3K inhibitor to enhance lapatinib activity was a general effect or more specific to the models with PI3K pathway alterations, we also tested the impact of the addition of GDC-0941 to lapatinib therapy in the OE33 cell line, a lapatinib-insensitive, ERBB2-amplified cell line, and found a much more modest effect $(\mathrm{CI}=0.8826)$ (Supplemental Figure 8 and Supplemental Table 6).

$M E T$ coamplification was associated with a lapatinib resistance that could be reverted by the addition of an MET-targeting drug. We next sought to investigate the potential for co-occurring genomic 
A

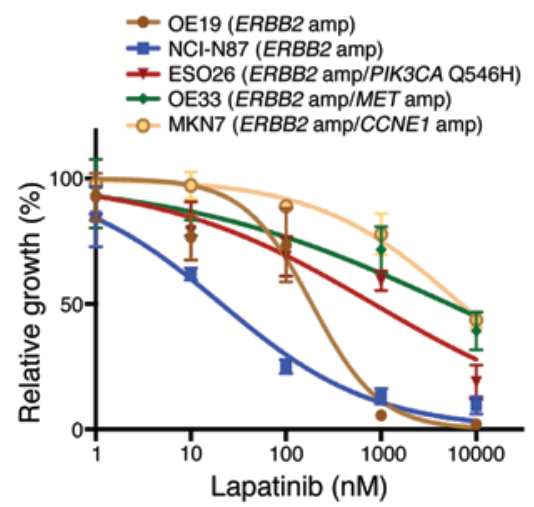

B

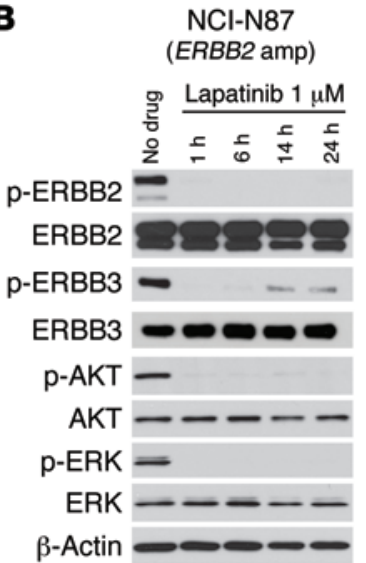

C

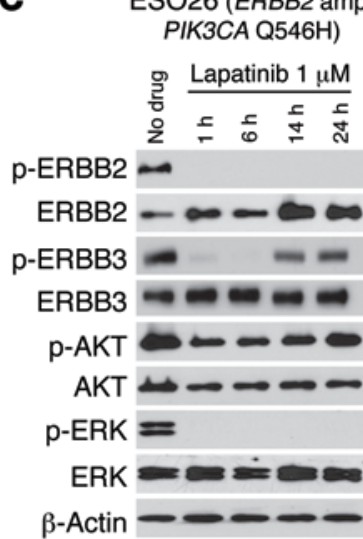

D

\section{OE33 (ERBB2} amp/MET amp)

o Lapatinib $1 \mu \mathrm{M}$ $\frac{5}{5} \frac{5}{5} \frac{7}{5}$ p-ERBB2 - - - -

ERBB2 p-ERBB3 ERBB3 $\square=\infty$

P-MET

MET

p-AKT

AKT

p-E

ERK

$\beta$-Actin
E

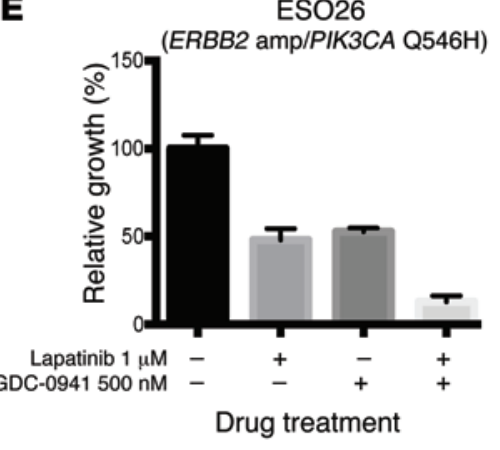

$\mathbf{F}$

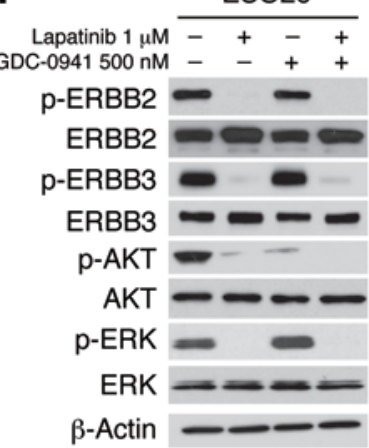

G

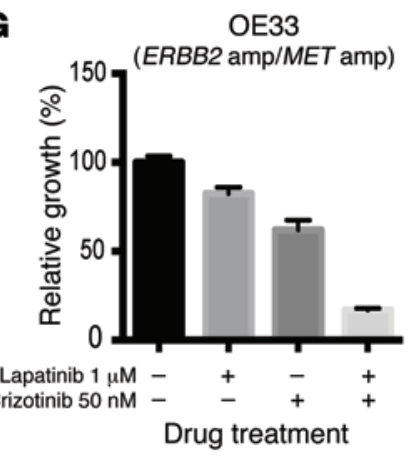

H

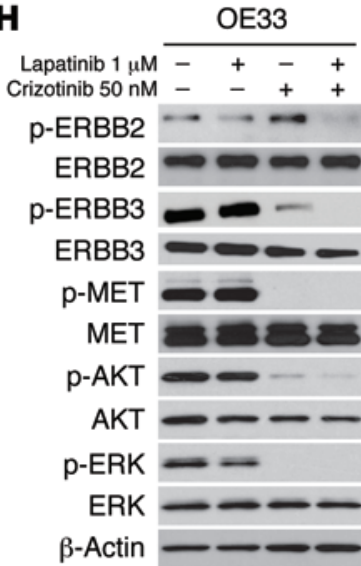

Figure 3. In vitro response to lapatinib of ERBB2-amplified GE adenocarcinoma cell-line models with endogenous PIK3CA mutation or MET amplification. (A) Lapatinib sensitivity curve for various ERBB2-amplified GE adenocarcinoma cell lines. Cell viability relative to vehicle-treated controls is shown. All experiments included 3 biological replicates, and each data point represents the mean \pm SEM. (B-D) Western blots showing the biochemical response to lapatinib in representative cell-line models. Cells were harvested at the indicated time points after treatment with $1 \mu \mathrm{M}$ lapatinib. (E and $\mathbf{G})$ In vitro growth inhibition by 2 small-molecule inhibitors either alone or in combination. Data represent drug treatment conditions and relative cell viability compared with that of vehicle-treated control. ( $\mathbf{F}$ and $\mathbf{H}$ ) Western blots showing the target engagement by treatment combinations. Cells were harvested 1 hour after drug treatment in the indicated combinations and doses. amp, amplification; $\mathrm{p}-$, phosphorylated.

amplifications of additional RTKs to impact the response to ERBB2 inhibition in GE adenocarcinomas, looking first at the kinase MET. The OE33 cell line, an ERBB2 and MET coamplified model (34), was notably insensitive to lapatinib (Figure 3A). Biochemically, ERBB2 phosphorylation was not decreased, despite treatment with $1 \mu \mathrm{M}$ lapatinib (Figure 3D), and MET was constitutively phosphorylated regardless of lapatinib treatment. Upon the combined treatment of the MET inhibitor crizotinib (35) with lapatinib, we found that the growth of OE33 was dramatically inhibited compared with that seen with either drug alone (Figure $3 \mathrm{G}$ ). Formal synergy testing of lapatinib and crizotinib in the OE33 cell line model revealed a CI value of 0.2816 , which was strongly synergistic (Supplemental Table 6). Moreover, ERBB2 phosphorylation disappeared only upon treatment with both drugs (Figure $3 \mathrm{H}$ ). To explain the potential mechanism by which MET could influence ERBB2 phosphorylation, we performed coimmunoprecipitation experiments in the OE33 model, revealing the physical interaction of MET and ERBB2 and sug- gesting direct phosphorylation of ERBB2 by constitutively active MET (Supplemental Figure 9).

Although trastuzumab treatment had little in vitro efficacy alone in OE33 cells, we observed a similar augmentation of response when it was combined with crizotinib treatment (Supplemental Figure 7B). We next tested crizotinib's ability to augment lapatinib sensitivity in cell lines without MET amplification, ESO26, or MKN7. Unlike in the OE33 cell line, MET inhibition did not appreciably impact lapatinib sensitivity in the ESO26 or MKN7 cell lines (Supplemental Figure 8). These results suggest that identification of secondary kinase amplifications that co-occur with $E R B B 2$ amplification may identify patients in whom dual-inhibitor strategies may be clinically efficacious.

Expression of activating PIK3CA mutants was required and was sufficient to induce lapatinib resistance in an ERBB2-amplified, lapatinib-sensitive cell-line model. To further investigate the causal role of PIK3CA mutation in drug resistance, we generated an isogenic cell-line model from the originally highly drug-sensitive NCI-N87 

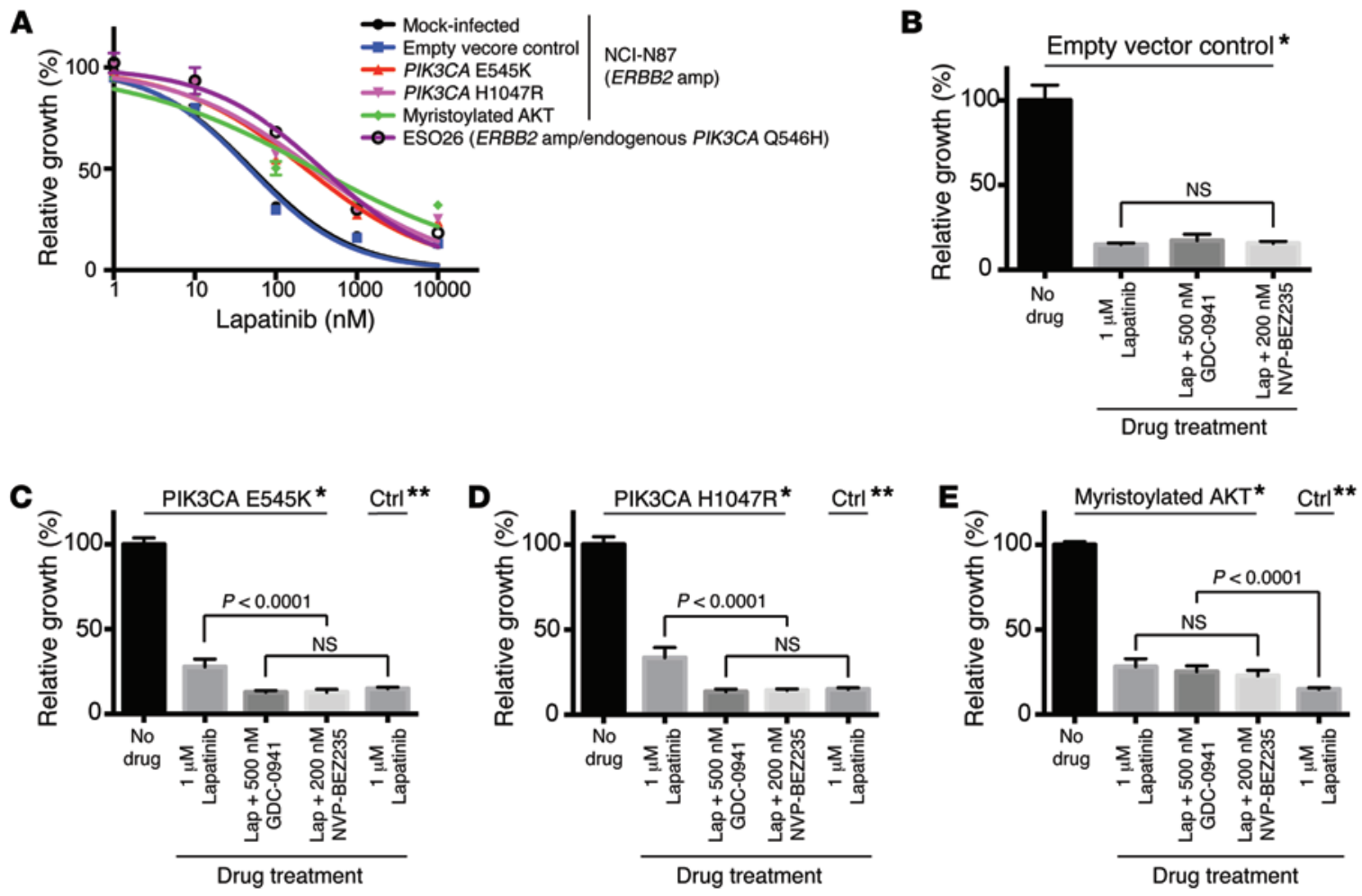

Figure 4. In vitro modeling of lapatinib resistance by exogenous expression of constructs that can activate the PI3K pathway in lapatinib-sensitive NCI-N87 cells. (A) Lapatinib sensitivity curves of NCI-N87 cells infected with the indicated constructs as well as those with mock or empty vector infection. Lapatinib sensitivity of ESO26, an ERBB2-amplified esophageal adenocarcinoma cell line with endogenous PIK3CA mutation (Q546H), is also shown. All data points are expressed as cell viability relative to that of vehicle-treated controls. (B-E) Growth inhibition of NCI-N87 cells with or without the introduction of mutant constructs, which activate the PI3K pathway, following treatment with lapatinib alone or in combination with GDC-0941 and NVP-BEZ235. Cell viability of empty control vector-infected NCI-N87 cells treated with lapatinib is shown in each panel as a baseline (C-E). P values were calculated by 1-way ANOVA. ${ }^{*} \mathrm{NCl}-\mathrm{N} 87$ cells infected with retrovirus containing each construct; ${ }^{* *}$ control (Ctrl) NCI-N87 cells infected with empty vector control. Lap, lapatinib.

cells into which we retrovirally transduced PIK3CA-mutant or constitutively active myristolyated AKT constructs. NCI-N87 cells expressing PIK3CA mutants or activated AKT shifted in their lapatinib sensitivity to levels equivalent to those of the ESO26 cells (Figure 4A). We found that the lapatinib resistance conferred by PIK3CA mutations was abolished by the combined treatment with either the PI3K-targeting drug GDC-0941 or the dual-PI3K/ mTOR inhibitor NVP-BEZ235 (Figure 4, C and D). The addition of these PI3K-targeting drugs did not augment the lapatinib effects in control NCI-N87 cells, implying that lapatinib could sufficiently inhibit the pathway in these cells (Figure 4B). However, the lapatinib resistance conferred by a constitutively active AKT was not affected by PI3K-targeting drugs, as would be expected, given that AKT is downstream of PI3K (Figure 4E). Taken together, PIK3CA mutations significantly reduced sensitivity to ERBB2-targeting agents in ERBB2-amplified GE cell line models, and sensitivity could be restored by combining PI3K and ERBB2 inhibition.

Coamplification of cell-cycle mediators reduces the responsiveness of GE adenocarcinomas to ERBB2 inhibition. A striking set of co-occurring oncogenic alterations was the frequent amplification of cellcycle mediators, namely CDK6, CCND1, and CCNE1, in ERBB2amplified GE cancers. Among these, amplifications of CCNE1 were notable, given both their frequency ( $24 \%$ of cases) and their recently reported role as an etiology of trastuzumab resistance in breast cancer (15). CCNE1 and CCND1, which encode cyclin E1 and cyclin D1, respectively, act by activating cyclin-dependent kinases to phosphorylate $\mathrm{Rb}$ and promote cell-cycle progression. Our analysis of genomic data from the 62 ERBB2-amplified GE tumors with the GISTIC tool revealed a highly significant $\left(\mathrm{q}=2 \times 10^{-16}\right)$ amplification occurring on chromosome 19q12. The peak of amplification identified by GISTIC included only 1 gene, CCNE1 (Supplemental Table 1). Focal amplifications of CCNE1 have been seen in genomic studies of breast cancer and uterine serous cancer (36-38).

Cyclin E1 complexes with the cell-cycle kinase CDK2 to phosphorylate $\mathrm{Rb}$ and promote the cell cycle. In breast cancer, acquired CCNE1 amplification is reported to induce trastuzumab resistance, which can be reversed by CDK2 blockade (15). We investigated drug sensitivity in the CCNE1- and ERBB2-coamplified gastric cancer cell line MKN7, finding it to be relatively insensitive to monotherapy with either lapatinib (Figure 5A) or the CDK1/2 inhibitor AZD5438 (Figure 5B). However, the addition of AZD5438 augmented the antiproliferative impact of lapatinib at a dose of $500 \mathrm{nM}$, which is able to effectively inhibit CDK2, as determined by inhibition of Rb phosphorylation (Figure 5C). Synergistic testing of lapatinib and AZD5438 revealed a CI value of 0.3489 in the MKN7 cell line (Supplemental Table 6). We also tested the addition of AZD5438 to lapatinib sensitivity in OE33 and ESO26 and found no similar evidence of synergy (Supplemental Table 6 and Supplemental Figure 8), thus suggesting that the ability of CDK2 inhibition to synergistically augment ERBB2 inhibition is 
A

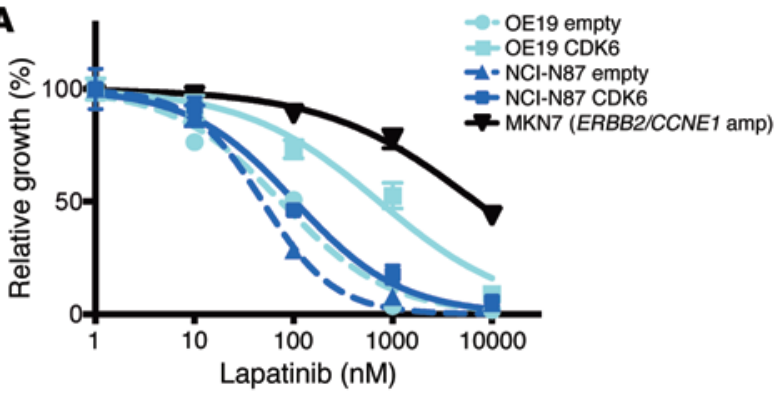

B

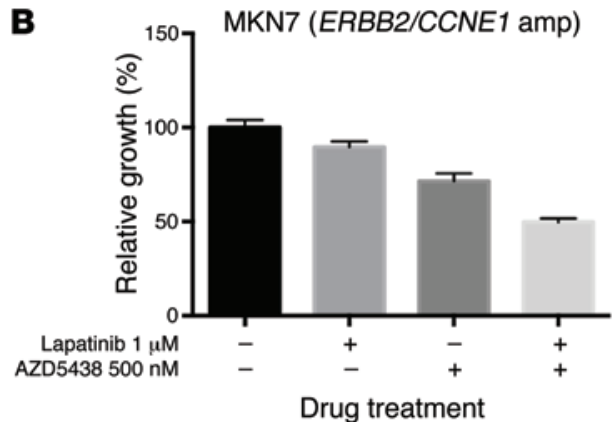

C

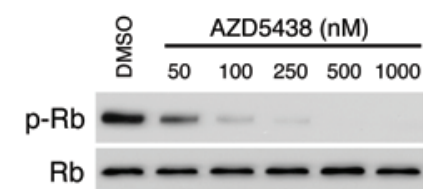

$\mathbf{F}$

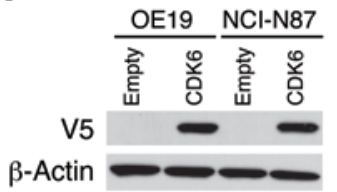

D

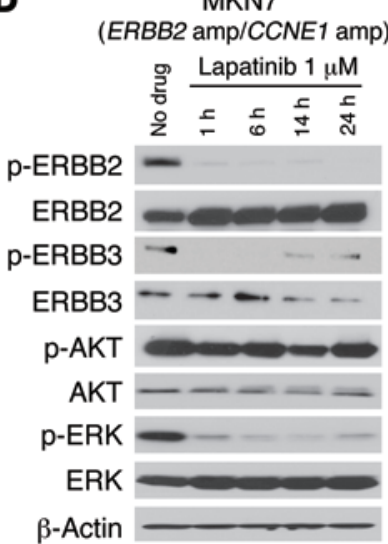

$\mathbf{E}$

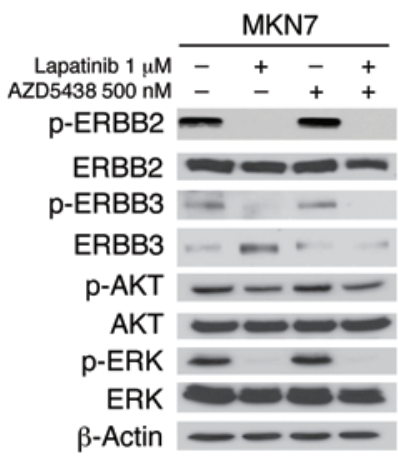

G

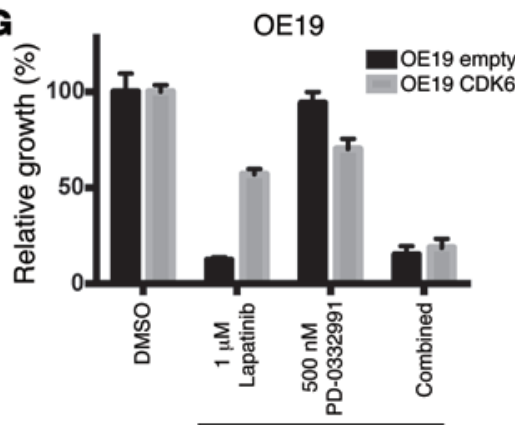

Drug treatment
H

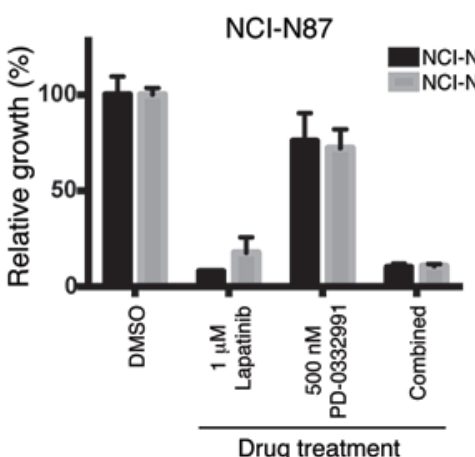

Figure 5. In vitro evidence for the role of the overexpression or amplification of cell-cycle-related genes in ERBB2-amplified GE cell lines. (A) In vitro lapatinib sensitivity curves for various ERBB2-amplified GE cell lines with or without concomitant overexpression of CCNE1 or CDK6. (B) Growth inhibition of ERBB2/CCNE1-coamplified gastric cancer cell line MKN7 by either lapatinib or AZD5438 alone or by a combination of both. All data are expressed as the percentage of growth relative to that of vehicle-treated control cells. (C) Western blot for $\mathrm{p}-\mathrm{Rb}$ and total Rb in MKN7 cells treated with increasing concentrations of AZD5438. Cells were harvested for protein extraction 6 hours after drug treatment. (D) Western blots showing MKN7 response to lapatinib at multiple time points. (E) Signaling responses of MKN7 cells to lapatinib alone or in combination with AZD5438. Cells were harvested 1 hour after each treatment. (F) Verification of CDK6 protein expression in our lentiviral expression system by Western blotting. Cells were harvested after blasticidin selection. (G and $\mathbf{H})$ Growth inhibition of OE19 (G) and NCI-N87 (H) cells with or without exogenous CDK6 expression by either lapatinib or palbociclib (PD0332991) alone or by a combination of both. All cell viability data are expressed as the percentage of growth relative to that of vehicle-treated control cells.

not a general feature of these cancers, but is perhaps specific to those tumors with CCNE1 amplification. However, we found that the insensitivity to lapatinib monotherapy and its reversal upon combined CDK2 blockade did not correlate with the phosphorylation of downstream signaling mediators such as AKT and ERK (Figure 5, D and E).

Beyond CCNE1 amplification, we also noted amplification of CCND1 and CDK6. Cyclin D1 acts with CDK4 and CDK6 to phosphorylate $\mathrm{Rb}$ and promote cell-cycle progression. Therefore, analogous to the use of CDK2 inhibitors in the setting of pathologic overexpression of cyclin E1, we hypothesized that the presence of cyclin D1 or CDK6 amplification/overexpression in the setting of ERBB2 amplification could mark tumors in which CDK4/6 inhibitors enhance the effects of ERBB2 inhibition. Although we lacked models with preexisting amplification of CDK6 or CCND1, we engineered the lapatinib-sensitive cell lines OE19 and NCI-N87 with ectopic CDK6 (or GFP control) (Figure 5F) and found CDK6induced lapatinib resistance (Figure $5 \mathrm{~A}$ ). The CDK4/6 inhibitor palbociclib (PD-0332991) did not have marked monotherapeutic effects but abrogated the impact of CDK6-induced resistance to 
A
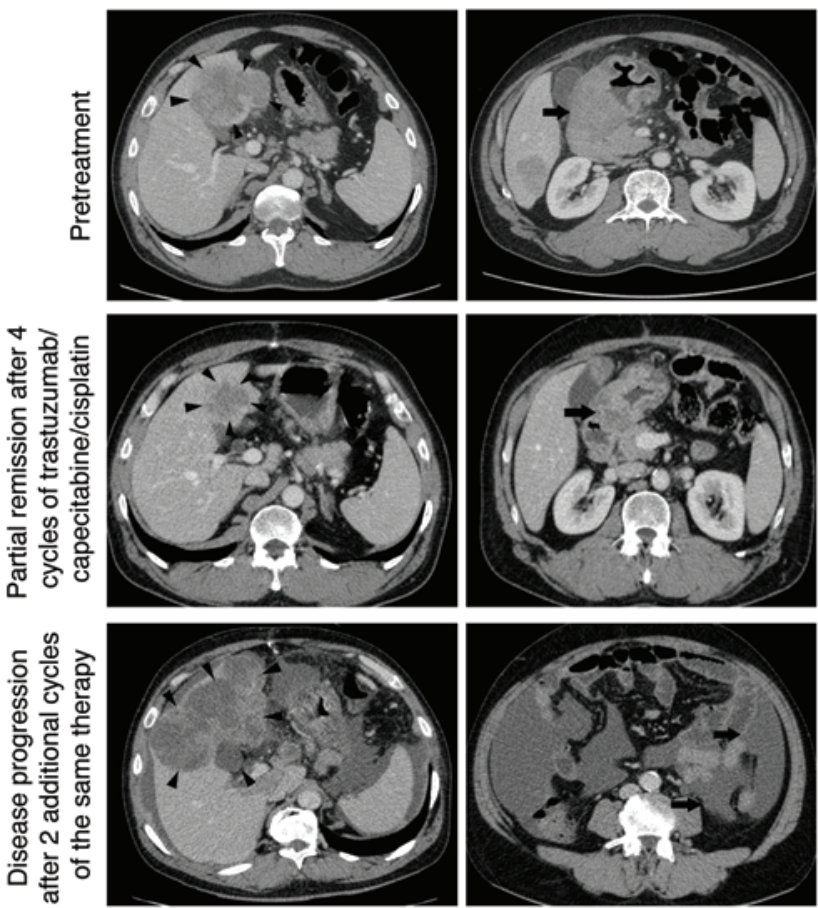

B
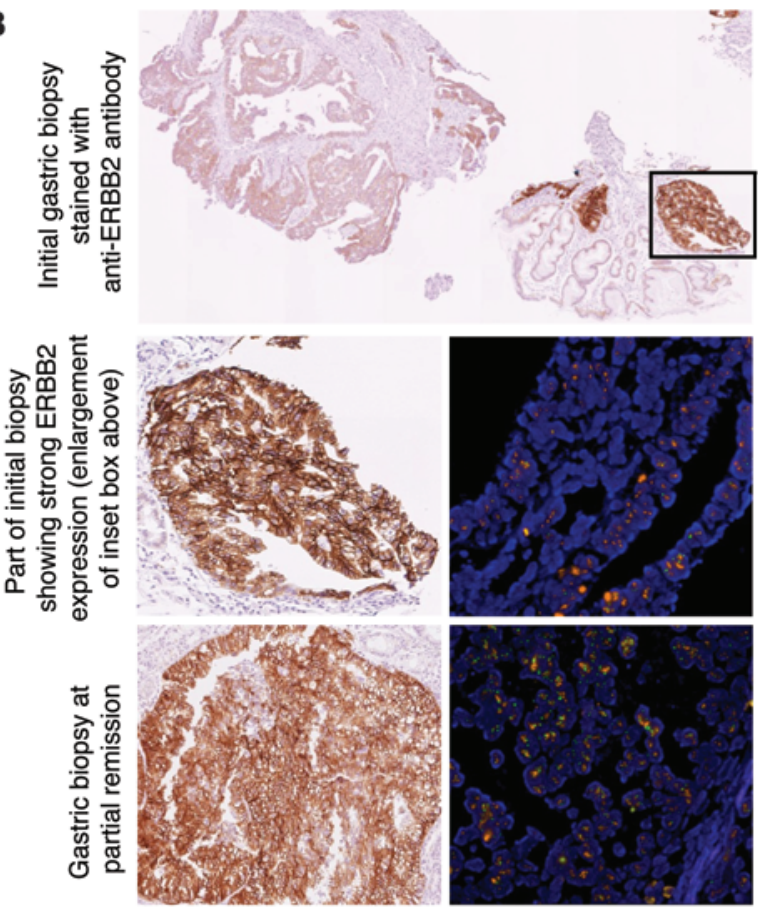

C

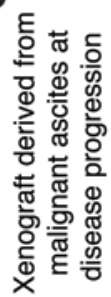

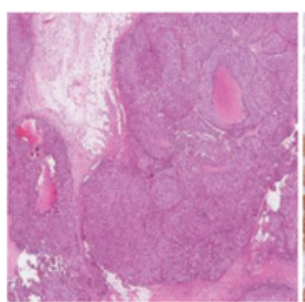

$H \& E$

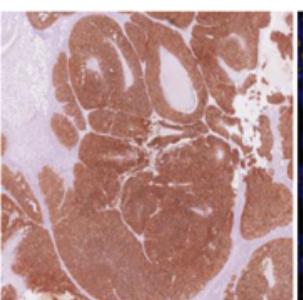

ERBB2

immunohistochemistry

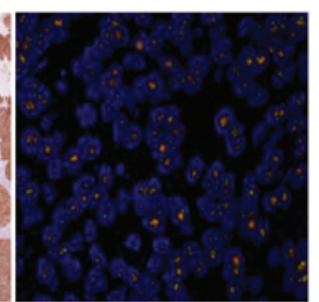

ERBB2 FISH

orange: ERBB2 green: CEP17

D

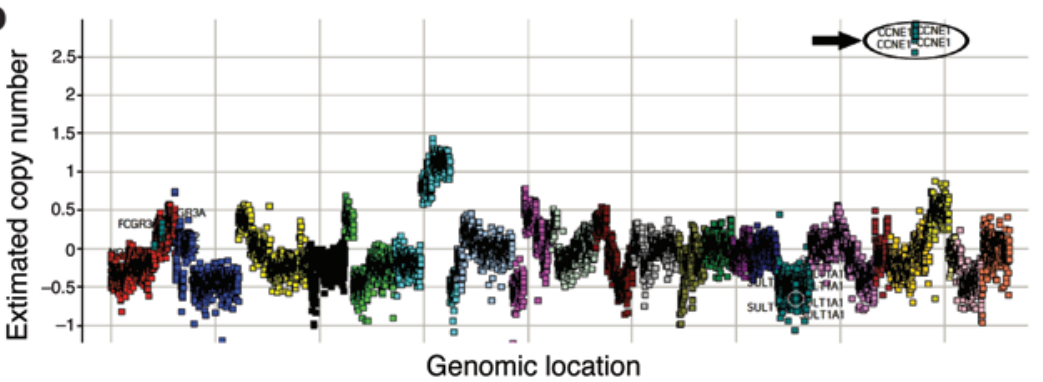

E

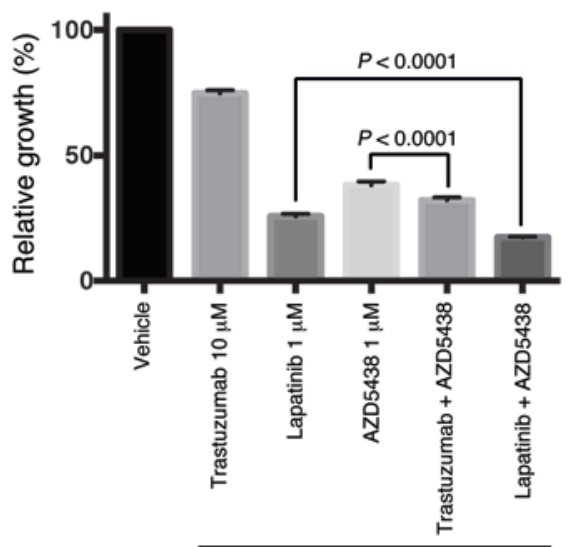

Drug treatment

Figure 6. Impact of CCNE1 amplification on drug response in an ERBB2-amplified gastric cancer patient. (A) CT images during treatment course. Initial liver metastasis (upper left, arrowheads) and primary gastric tumor (upper right, arrow) show a significant size reduction after 4 cycles of trastuzumab-containing chemotherapy (middle panels). During 2 additional cycles, the liver metastasis (lower left, arrowheads) grew and ascites (lower right, arrow) developed. (B) Immunohistochemistry of ERBB2 (left panels and top right panel; original magnification, $\times 200$; inset, $\times 40$ ) and FISH of ERBB2 locus (right, middle and bottom panels: original magnification, $\times 1,000$ ). ERBB2 protein expression in pretreatment gastric tumor biopsy is shown as brown membranous staining. ERBB2 amplification in the pretreatment gastric tumor cells with strong ERBB2 protein expression (middle right panel; orange: ERBB2, green: CEP17). Gastric biopsy at the disease progression stage exhibited homogeneously strong ERBB2 expression and ERBB2 amplification (bottom right panel). (C) Xenograft derived from malignant ascites at the disease progression stage. Tumor nests were seen in H\&E staining (left; original magnification, $\times 40$ ). Tumor cells showed strong ERBB2 protein expression (middle; original magnification, $\times 40$ ) and ERBB2 amplification (right; original magnification, $\times 1,000)$. (D) Estimated copy-number plot from targeted exome-sequencing data of xenograft derived from malignant ascites. Note that the CCNE1 locus is amplified. (E) In vitro growth inhibition of malignant ascites-derived gastric cancer cells treated with small-molecule or antibody ERBB2 inhibitors with or without CDK2 inhibition. 
ERBB2 inhibition (Figure 5, G and H). Unlike with ERBB2 ${ }^{+}$models with coexisting $M E T$ or PIK3CA alterations, neither overexpression of CDK6 nor inhibition of CDK6 altered the effects of lapatinib upon phosphorylation of AKT (Supplemental Figure 10). Together with the results from the MKN7 cell line, these data support the potential for testing the addition of targeted agents inhibiting cellcycle progression to augment the impact of ERBB2-directed therapy in select patients with ERBB2 ${ }^{+} \mathrm{GE}$ adenocarcinomas.

Effect of CDK2 inhibitor therapy on patient-derived cells of ERBB2/CCNE1 coamplified gastric cancer. We also sought to supplement our in vitro studies of ERBB2 resistance by evaluating a patient with metastatic $\mathrm{ERBB} 2^{+}$gastric cancer who achieved partial response to trastuzumab/capecitabine/cisplatin but whose disease subsequently progressed during trastuzumab treatment (Figure 6A). Tumor samples were available from both the initial primary tumor and a liver metastasis at the disease progression stage. While we initially intended to evaluate the acquired resistance of ERBB2 ${ }^{+}$ GE adenocarcinoma, detailed analysis suggested that despite the apparent clinical response, this particular tumor may paradoxically have harbored de novo resistance of the ERBB2 $2^{+}$tumor to ERBB2containing therapy. Pathologic review of the pretreatment sample revealed that the ERBB2-amplified section was only a small subset of the larger primary tumor. In contrast to our expectations, the tumor that emerged following therapy showed homogeneous ERBB2 positivity, indicating that the ERBB2-amplified subpopulation emerged during trastuzumab therapy (Figure 6, B and C) due to intrinsic resistance to trastuzumab-containing therapy.

Genomic testing revealed the presence of ERBB2 and CCNE1 coamplification in both the pretreatment sample and the resistant metastatic lesion (Figure 6, D and E). To evaluate the hypothesis that the CCNE1 amplification attenuated ERBB2 inhibitor sensitivity, malignant cells from ascites taken at the time of disease progression were cultured for drug sensitivity testing. Indeed, although in vitro testing of this culture showed moderate sensitivity to lapatinib, treatment with the CDK1/2 inhibitor AZD5438 was able to augment the antiproliferative impact of lapatinib (Figure $6 \mathrm{~F}$ ), consistent with our earlier results in the CCNE1/ERBB2coamplified cell line MKN7. This model was insensitive to trastuzumab therapy in vitro. However, we found that trastuzumab was able to modestly increase the sensitivity of this model to CDK2 inhibition. While there could be many alternative etiologies of therapy failure in any given patient, this example offers initial clinical support for our hypothesis that CCNE1 amplification can attenuate ERBB2 inhibitor sensitivity and that emerging CDK2directed agents should be evaluated in such patients as a potential means to enhance response to therapy.

This clinical example also raised another possible factor that could lead to the presence of multiple distinct activated oncogenes in ERBB2-amplified samples: that is, intratumor heterogeneity. In this patient, the CCNE1 amplification was found in both the region of the tumor with ERBB2 amplification and in the ERBB2negative region. To follow the observation from this patient, we investigated ERBB2 and cyclin E1 protein expression across additional $E R B B 2^{+} \mathrm{GE}$ adenocarcinomas. We evaluated 23 gastric cancers with strong (3+) ERBB2 expression and found 10 cases (43.5\%) with cyclin E1 protein overexpression (Supplemental Figure 11). Eight of the 10 cases showed homogeneous expression of both cyclin E1 and ERBB2, demonstrating that these oncoproteins are frequently coexpressed in the same tumor cells, consistent with the potential for cyclin E1 overexpression to be an etiology of resistance to ERBB2-directed therapy.

Overexpression of EGFR with ERBB2 may impart sensitivity to dual EGFR-ERBB2 inhibitors. We next explored the observation from our genomic analysis that tumors with $E R B B 2$ amplification co-occurred with EGFR amplification in GE adenocarcinomas more commonly than in breast cancer. EGFR is known to play an important role in gut epithelial homeostasis $(39,40)$, perhaps providing some rationale for the greater amplification of EGFR in gut-derived cancer. Because overexpression of EGFR has been implicated in trastuzumab resistance in ERBB2-amplified breast cancers $(17,18,41)$, we sought to investigate the expression of EGFR in ERBB2-amplified GE adenocarcinoma compared with that in breast cancer. We first queried mRNA expression of $E G F R$, $E R B B 2$, and $E R B B 3$ in primary ERBB2-amplified breast and GE adenocarcinomas using TCGA data (32). While ERBB2 levels were similar in the 2 populations, ERBB3 mRNA expression was significantly higher in the breast cancers $(P<0.0001)$, and EGFR mRNA expression showed a trend toward higher levels in gastric tumors $(P=0.157)$ (Figure 7D). Further analysis of EGFR expression relative to the genomic status of EGFR within TCGA data revealed that in addition to cases with genomic alterations of $E G F R$, further cases also harbored elevated expression (Supplemental Figure 12), suggesting that EGFR plays a role in GE adenocarcinoma, even in cases lacking genomic alterations of EGFR. Similarly, immunohistochemical analysis of a separate sample set showed a trend toward more frequent EGFR protein expression in ERBB2-amplified GE adenocarcinoma ( 7 of $12,58.3 \%$ ) than in breast cancers (12 of 32, 31.3\%) (Figure 7E). Together, these data suggested that in $\mathrm{ERBB}^{+} \mathrm{GE}$ adenocarcinoma, coexisting EGFR expression may commonly occur, raising the question of whether the presence of this co-occurring event also has therapeutic implications.

We next evaluated the hypothesis that EGFR expression impacts the sensitivity of ERBB2 ${ }^{+} \mathrm{GE}$ adenocarcinomas to ERBB2 inhibition. We noted that the pattern of sensitivity of the GE adenocarcinoma cells to trastuzumab and lapatinib was discrepant from that of breast cancer cells (Figure 7, A and B). We observed that OE19 and NCI-N87 were sensitive to lapatinib but more resistant to trastuzumab, whereas breast cancer cells such as SK-BR3 and ZR75-30 were sensitive to trastuzumab but were less sensitive to lapatinib. BT474 cells were sensitive to both drugs. The discrepancy in drug sensitivity for NCI-N87 is supported by recent xenograft data (42). We evaluated the expression of ERBB2, EGFR, and ERBB3 among these models. Indeed, although OE19 and NCI-N87 cells did not harbor genomic alterations of EGFR, they had more abundant phosphorylated and total EGFR than did BT474 and ZR75-30 cells (Figure 7C). In contrast, phosphorylated and total ERBB3 was more abundant in breast cancer cells. Those differences in EGFR and ERBB3 abundances were clearly associated with the relative amounts of EGFR:ERBB2 or ERBB3:ERBB2 heterodimeric complexes, as determined by coimmunoprecipitation of ERBB2 from cell-line models (Supplemental Figure 13).

We hypothesized that with higher EGFR expression, EGFR blockade may augment the effect of trastuzumab, which targets only ERBB2. Indeed, in OE19, NCI-N87, and SK-BR3, the addi- 
A

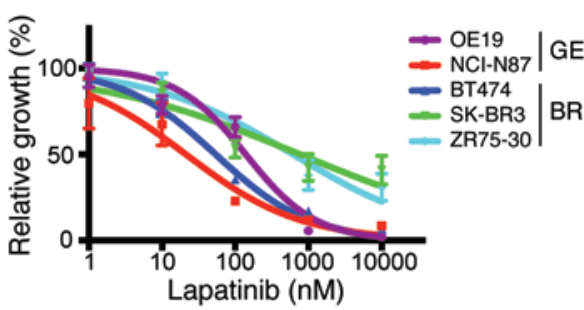

B

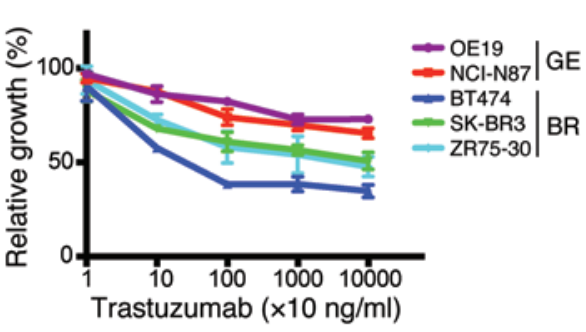

D
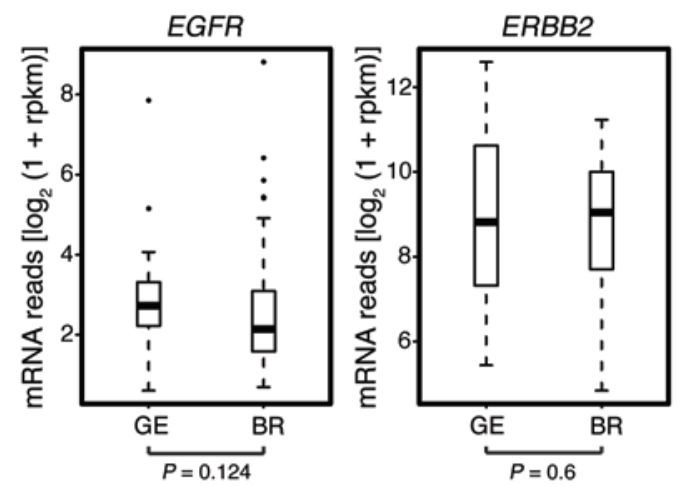

C
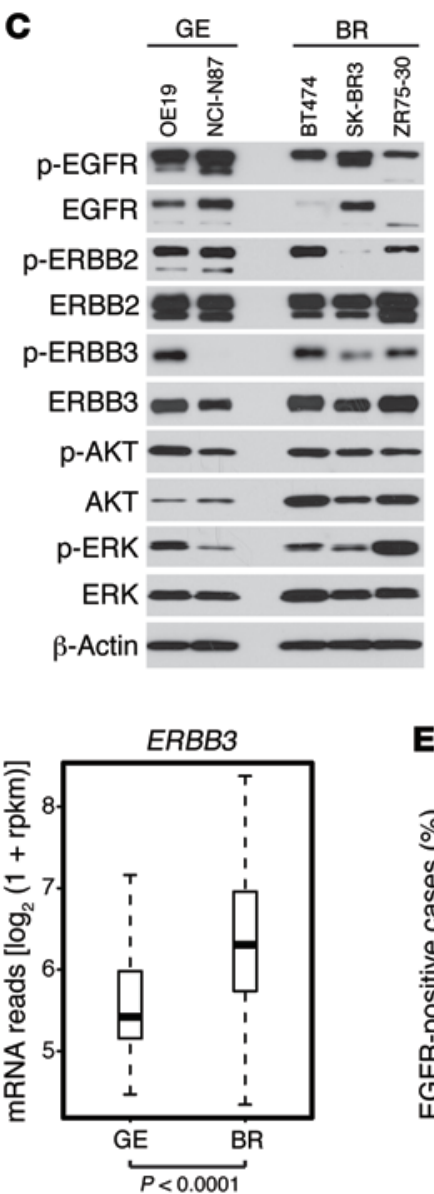

E

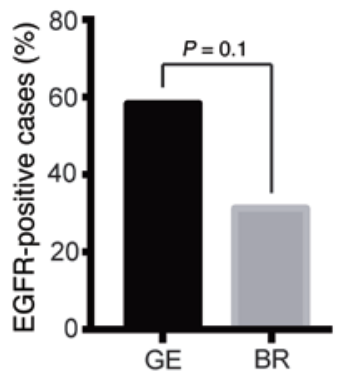

$\mathbf{F}$

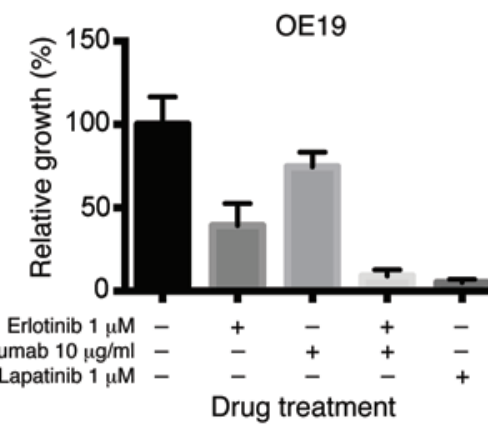

\begin{abstract}
G
\end{abstract}

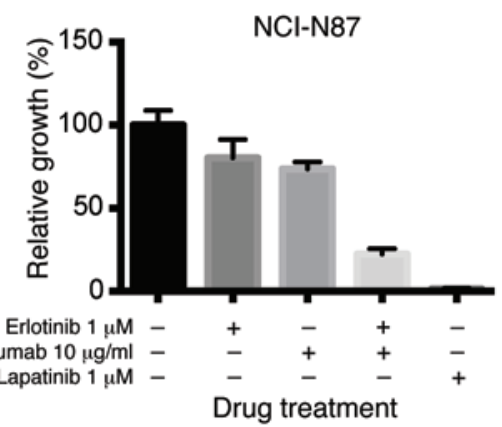

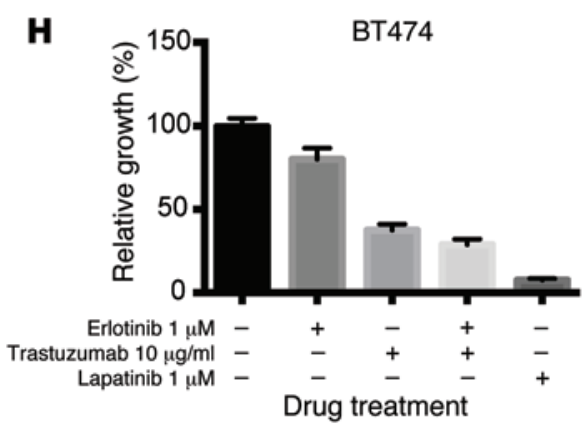
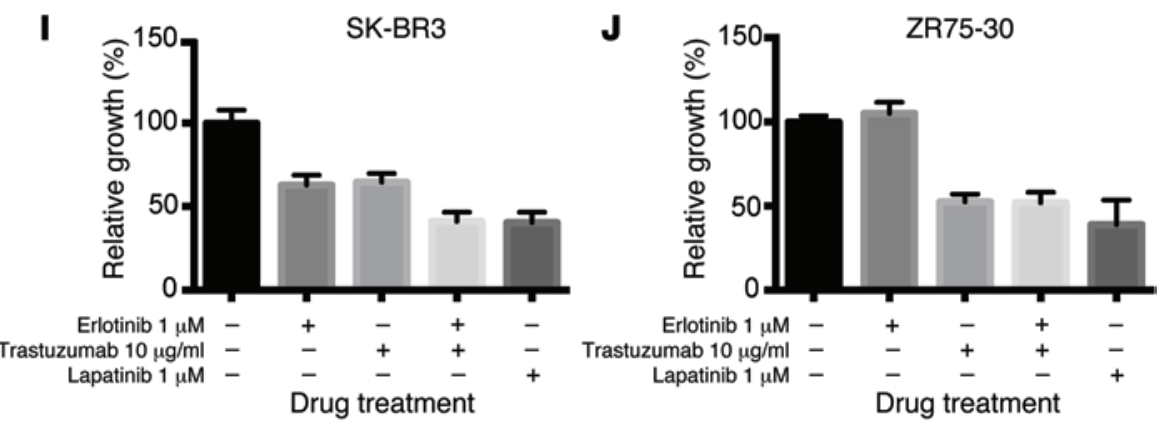
Figure 7. Impact of EGFR expression on the responsiveness of ERBB2amplified GE adenocarcinoma and breast cancer cell lines to trastuzumab treatment in vitro. (A and B) Sensitivity of ERBB2-amplified GE adenocarcinoma and breast cancer cell lines to lapatinib or trastuzumab. Cell viability was measured 72 hours after lapatinib treatment and 120 hours after trastuzumab treatment. (C) Western blot showing the expression of phosphorylated and total ERBB members as well as their downstream effectors across a panel of ERBB2-amplified GE adenocarcinoma and breast cancer cell lines. Protein was harvested 24 hours after plating at the desired densities. Cells were kept in full serum-containing medium. (Note, compared with the NCI-N87 p-ERBB3 immunoblot in Figure 3 showing evidence of $\mathrm{p}$-ERBB3, this NCI-N87 blot does not show the p-ERBB3 signal because a shorter exposure was applied for better comparison with other cell lines.) (D) Comparison of ERBB member mRNA expression between ERBB2-amplified GE adenocarcinoma and breast cancer cases using RNA-sequencing data from TCGA datasets. $P$ values were calculated by 2 -tailed Student's $t$ test. Boundaries of the boxes represent the first and third quartiles; horizontal line in each box represents the median; whiskers represent the lowest and highest values within 1.5 times the interquartile range from the lower and upper quartiles; outliers represent any value beyond that of the whiskers. $\mathrm{GE}, n=28 ; \mathrm{BR}, n=113$. rpkm, reads assigned per kilobase of target per million mapped reads. (E) Frequency of EGFR protein expression in ERBB2-amplified GE adenocarcinoma and breast cancer cases. Immunohistochemical analysis showing the percentage of cases with membranous EGFR staining in more than $10 \%$ of tumor cells. $\mathrm{GE}, n=12$; BR, $n=32$. $P$ value was obtained by $\chi^{2}$ test. $(F-J)$ In vitro growth inhibition of various ERBB2-amplified GE adenocarcinoma and breast cancer cell lines following treatment with erlotinib, trastuzumab, or both. Cell viability upon lapatinib treatment is shown for comparison. All cell viabilities were measured 5 days after drug treatment.

tion of erlotinib increased the antiproliferative effect of trastuzumab (Figure 7, F-J). Although the phosphorylation of EGFR or ERBB2 was not effectively inhibited by trastuzumab or erlotinib in those cells, it was further inhibited by the combination of both (Supplemental Figure 14). By contrast in BT474 and ZR75-30, the addition of erlotinib to trastuzumab therapy had little impact, thus reducing the concern that erlotinib effects in EGFR-positive lines stem from its potential direct inhibition of ERBB2 (43). These data suggest that ERBB2-positive GE adenocarcinoma patients with expression or genomic activation of EGFR may benefit from dual ERBB2 and EGFR inhibition.

\section{Discussion}

Through comprehensive genomic characterization and functional validation, we demonstrated that more than a half of the ERBB2-amplified GE adenocarcinomas had additional oncogenic alterations that could potentially hamper the growth-inhibitory effect of ERBB2-targeting drugs. Among the oncogenic alterations, we showed that amplifications of cell-cycle-related genes such as CCNE1 and CDK6, PI3K pathway activation by activating PIK3CA mutations, and amplification of MET conferred resistance to ERBB2-targeting drug in in vitro ERBB2-amplified cell-line models. We also demonstrated that the combined treatment of ERBB2-amplified cells having those secondary oncogenic alterations with drugs targeting the secondary oncogene enhanced the inhibition of cell growth. Finally, we present new data suggesting that in the setting of ERBB2 amplification, elevated EGFR expression identifies tumors that would be more effectively treated with combined ERBB2 and EGFR small-molecule inhibitors than with antibodies that target ERBB2 alone.
Secondary oncogenic events spanned RTKs, cell-cycle mediators, and the PI3K signaling pathway. It is likely that as more tumors are genomically profiled, we will identify additional co-occurring alterations. Although it has not been fully established whether CCNE1 is itself an oncogene, in breast cancers, amplification and overexpression of CCNE1 has been implicated in the resistance to trastuzumab treatment (15). Furthermore, PI3K pathway activation by activating PIK3CA gene mutations or by inactivation of PTEN has been reported to confer resistance to ERBB2-directed therapies $(12,44)$. In this report, we show for the first time to our knowledge that amplifications of cell-cycle-related genes such as CCNE1 and CDK6 and the persistent activation of the PI3K pathway coexist in considerable proportions of $E R B B 2$-amplified GE adenocarcinomas (40.5\% and $11.9 \%$, respectively). Also, we present what we believe to be novel functional evidence that these genomic alterations contribute to attenuated sensitivity of in vitro ERBB2-amplified GE adenocarcinoma cell-line models to ERBB2 inhibition.

$M E T$ amplification and ERBB2 amplification had been thought to be mutually exclusive based on analyses of smaller datasets (29). However, with the unprecedented size of this analysis, we identified the frequent co-occurrence of $M E T$ amplification in the setting of ERBB2 amplification. Although the role of MET in resistance to ERBB2-directed therapies has been reported in gastric cancer (45), that report evaluated only the effect of the exogenous ligand HGF, not the amplification of MET. Our analysis also revealed that $E R B B 2^{+} \mathrm{GE}$ adenocarcinomas recurrently harbor EGFR coamplification and that EGFR may be overexpressed in tumors lacking EGFR genomic aberrations. Our data suggest that for those ERBB2amplified GE adenocarcinomas with abundant EGFR expression, existing dual EGFR/ERBB2-directed therapies may be more effective. However, our hypothesis must be considered in light of recent clinical data on the use of EGFR and dual EGFR/ERBB2 inhibitors to treat $\mathrm{ERBB}^{+} \mathrm{GE}$ adenocarcinomas. Use of an EGFR inhibitor as a single agent or in combination with chemotherapy has not been proven to be clinically effective $(46,47)$. The results in 10 patients with ERBB2-amplified GE adenocarcinoma who received the single agent afatinib, an irreversible dual EGFR/ERBB2 inhibitor, were disappointing (48). Furthermore, the systematic use of lapatinib in addition to chemotherapy did not lead to a statistically significant improvement in the survival rates of ERBB2 $2^{+}$patients when given either as frontline therapy in the phase III LOGiC trial or as a second-line treatment in the phase III TyTAN trial $(9,49)$. However, within the TyTAN trial, the subgroup with $3^{+}$ERBB2 status, as demonstrated by FISH, showed an increase in survival with receipt of lapatinib following failure of trastuzumab (49). One potential limitation in our study is that only limited cell-line models were available. Therefore, future extended analysis using primary human GE adenocarcinoma tissue or patient-derived xenografts with distinct combinations of targets will be necessary to further validate our hypotheses. Taken together, it will be important to further evaluate the secondary genomic and molecular features of tumors in trials of distinct ERBB2 inhibitors to determine whether specific features, such as EGFR expression, identify patients who have distinct responses to specific classes of agents and those who have a broader poor response to ERBB2-directed therapy.

These observations also raise other questions that will need to be addressed in future studies. In some cases, tumors can har- 
bor more than 2 actionable mutations. In these situations, it will be important to evaluate which secondary targets should be prioritized and develop combination strategies that allow patients to tolerate dosing of multiple inhibitors. Targeting multiple driver events within the same tumor will place a higher importance on patient selection for these studies and will also require efforts to not only measure drug target engagement, but to also validate that the drug combinations are effectively inhibiting the activity of downstream targets such as AKT and ERK. Studies will also be needed to address the question of genomic heterogeneity within GE cancers. Intratumoral heterogeneity of ERBB2 expression and amplification has been reported (50), but our data do not reveal whether those alterations co-occur in the same cells in tumors with multiple oncogenic alterations. Indeed, there may be cases in which secondary oncogenic events occur in subpopulations distinct from the population of cells with ERBB2 amplification. Even in the case of genomic heterogeneity, however, optimal therapy would likely require combinations of targeted therapies to attack distinct drivers of genomic alterations.

Prior to the introduction of trastuzumab combination therapy, systemic therapy for GE adenocarcinomas effectively treated all tumors as a single entity. The new paradigm by which ERBB2 status is used as a biomarker to select patients for trastuzumabcontaining therapy is a substantial advance. However, current efforts to identify the means of enhancing ERBB2-amplified GE adenocarcinoma therapy approach ERBB2-amplified GE adenocarcinomas as a single entity, with the goal of identifying optimal treatment for all patients with these tumors. Furthermore, these current approaches do not take into consideration the potential impact of oncogenes other than ERBB2 on the selection of optimal therapy. Indeed, many current trials for trastuzumab-refractory disease are focused on testing new, second-generation ERBB2directed therapies in the hope that augmented ERBB2 inhibition will be able to restore sensitivity. Based on our focused genomic analysis of ERBB2-amplified GE adenocarcinomas, we propose a new model, in which these ERBB2 ${ }^{+}$GE cancers can be further subdivided by the presence of other preexisting alterations that cooccur with $E R B B 2$ amplification. Our data suggest that the molecular subclassification of $E R B B 2$-amplified GE adenocarcinomas and the development of clinical trials testing a combination treatment according to the subclassification might significantly improve therapeutic outcomes for patients with this deadly disease.

\section{Methods}

Genomic characterization of human samples and cell-line models. To interrogate the significantly co-occurring oncogenic copy-number alterations in ERBB2-amplified cancers, we took 743 gastric cancer samples with SNP Array 6.0 data from the published dataset (26, 29). From this set, we identified 62 ERBB2-amplified cases, defined by normalized $\log _{2}$ (copy-number ratio of tumor/normal) of 0.9 or greater. Using the same criteria, we pulled 103 ERBB2-amplified breast cancer cases ( $11.2 \%)$ out of 916 patients (32). Then, we ran GISTIC 2.0 (51) on the copy-number data for each sample set. To identify co-occurring oncogenic mutations, we analyzed exomesequencing data on samples from our previously published studies (27) and samples from TCGA (30). We were able to collect 486 samples with exome-sequencing data, of which 42 (8.6\%) had evidence of ERBB2 amplification based on either matched SNP arrays or copy numbers inferred from exome data (27).

Genomic characterization of patient-derived tissue samples. We evaluated a patient with metastatic ERBB2-amplified gastric adenocarcinoma who had a partial response to the combination of trastuzumab, capecitabine, and cisplatin and whose disease subsequently progressed during trastuzumab treatment. Fresh-frozen tissue samples were obtained from an initial gastric biopsy and from malignant ascites at the disease progression stage. Genomic characterization was performed using a focused Illumina exome capture and sequencing platform as described previously (http://www.ncbi.nlm.nih.gov/ biosample/3106204) (52).

In vitro drug sensitivity assay. Cell viability was measured by the CellTiter-Glo Luminescent Cell Viability assay (Promega) according to the manufacturer's instructions. For small-molecule inhibitors, cells were allowed to grow for 3 days, and for trastuzumab, cells were grown 5 days after treatment. All experiments had 4 technical replicates and 3 biological replicates. Synergy testing was performed using the ChouTalalay method as previously described (53). Briefly, 5 different concentrations of each drug (0.25X, 0.5X, 1X, 2X, and 4X IC50) were given either alone or in combination, maintaining a constant ratio. Viability was then measured using CellTiter-Glo, and the results were analyzed using COMPUSYN (53).

Detection of EGFR:ERBB2 or ERBB3:ERBB2 heterodimers. To detect oligomerized ERBB family member proteins, we crosslinked protein in cultured cells with BS3 (bis[sulfosuccinimidyl] suberate) (Pierce Biotechnology) as described previously (54). Crosslinked cell cultures were harvested and immunoprecipitated with anti-ERBB2 antibody. Immunoprecipitates were probed with either anti-EGFR or anti-ERBB3 antibody.

Tissue microarray and immunohistochemistry. To evaluate the relative frequency of EGFR expression in ERBB2-amplified GE adenocarcinomas and in breast cancers, we used tissue microarrays that had matched copy-number information. ERBB2 overexpression and amplification were confirmed by immunohistochemistry and chromogen in situ hybridization (CISH) as described previously (55). We stained sections of tissue microarrays with anti-EGFR antibody (clone 2-18C9; Dako). EGFR staining was considered positive when more than $10 \%$ of the stained tumor cells showed unequivocal membranous staining.

To address the intratumoral heterogeneity in terms of ERBB2 and CCNE1 overexpression in the ERBB2-amplified GE adenocarcinoma, we performed immunohistochemistry with anti-ERBB2 (Novocastra) and CCNE1 (clone HE12; Thermo Fisher Scientific) on a collection of 23 human gastric cancers with strong (3+) ERBB2 expression.

Patient-derived tumor cells and drug treatment. Malignant cells were isolated from malignant ascites drained for therapeutic purposes. The cells were cultured in RPMI medium supplemented with 10\% FBS. Cell growth inhibition was determined by CellTiter 96 Aqueous One Solution assay (Promega) according to the manufacturer's protocol.

Statistics. Statistical analyses of somatic copy-number data were performed with GISTIC as detailed in the Supplemental Methods. For functional experiments, comparisons between experimental arms were performed by 2-tailed Student's $t$ tests, with $P$ values of less than 0.05 considered significant. For all figures displaying numerical data, the bar charts show the mean with error bars representing SEM.

Study approval. Cells derived from a single patient were collected with the patient's written informed consent and IRB approval of Sam- 
sung Medical Center (Seoul, Republic of Korea). All other studies used publicly available genomic datasets and established and commercially available cell-line models.

\section{Acknowledgments}

A.J. Bass was supported by the AGA-Funberburg Award in Gastric Cancer Biology, the Phi Beta Psi Sorority, and a Research Scholar Grant from the American Cancer Society. A.K. Rustgi, K.K. Wong, and A.J. Bass were supported by NIH grant P01 CA098101. A.K. Rustgi was also supported by NIH grants R01 DK060694, R01 DK056645, and P30 DK050306.

Address correspondence to: Adam Bass, Department of Medical Oncology, Dana-Farber Cancer Institute, 450 Brookline Avenue, Boston, Massachusetts 02215, USA. Phone: 617.632.2477; E-mail: adam_bass@dfci.harvard.edu.
1. Jemal A, Center MM, DeSantis C, Ward EM. Global patterns of cancer incidence and mortality rates and trends. Cancer Epidemiol Biomarkers Prev. 2010;19(8):1893-1907.

2. Holmes RS, Vaughan TL. Epidemiology and pathogenesis of esophageal cancer. Semin Radiat Oncol. 2007;17(1):2-9.

3. Druker BJ, et al. Effects of a selective inhibitor of the Abl tyrosine kinase on the growth of Bcr-Abl positive cells. Nat Med.1996;2(5):561-566.

4. Flaherty KT, et al. Inhibition of mutated, activated BRAF in metastatic melanoma. $N$ Engl J Med. 2010;363(9):809-819.

5. Lynch TJ, et al. Activating mutations in the epidermal growth factor receptor underlying responsiveness of non-small-cell lung cancer to gefitinib. N Engl JMed. 2004;350(21):2129-2139.

6. Paez JG, et al. EGFR mutations in lung cancer: correlation with clinical response to gefitinib therapy. Science. 2004;304(5676):1497-1500.

7. Slamon DJ, et al. Use of chemotherapy plus a monoclonal antibody against HER2 for metastatic breast cancer that overexpresses HER2. N Engl J Med. 2001;344(11):783-792.

8. Bang YJ, et al. Trastuzumab in combination with chemotherapy versus chemotherapy alone for treatment of HER2-positive advanced gastric or gastro-oesophageal junction cancer (ToGA): a phase 3, open-label, randomised controlled trial. Lancet. 2010;376(9742):687-697.

9. Hecht JR, et al. Lapatinib in combination with capecitabine plus oxaliplatin in HER2-positive advanced or metastatic gastric, esophageal, or gastroesophageal adenocarcinoma: The TRIO-013/LOGiC Trial [abstract]. JClin Oncol. 2013; (suppl 31):LBA4001.

10. Berns K, et al. A functional genetic approach identifies the PI3K pathway as a major determinant of trastuzumab resistance in breast cancer. Cancer Cell. 2007;12(4):395-402.

11. Dave B, et al. Loss of phosphatase and tensin homolog or phosphoinositol-3 kinase activation and response to trastuzumab or lapatinib in human epidermal growth factor receptor 2-overexpressing locally advanced breast cancers. J Clin Oncol. 2011;29(2):166-173.

12. Eichhorn PJ, et al. Phosphatidylinositol 3-kinase hyperactivation results in lapatinib resistance that is reversed by the mTOR/phosphatidylinositol 3-kinase inhibitor NVP-BEZ235. Cancer Res. 2008;68(22):9221-9230.

13. Garrett JT, et al. Transcriptional and posttranslational up-regulation of HER3 (ErbB3) compensates for inhibition of the HER2 tyrosine kinase. Proc Natl Acad Sci U S A. 2011;108(12):5021-5026.

14. Junttila TT, et al. Ligand-independent HER2/
HER3/PI3K complex is disrupted by trastuzumab and is effectively inhibited by the PI3K inhibitor GDC-0941. Cancer Cell. 2009;15(5):429-440.

15. Scaltriti M, et al. Cyclin E amplification/overexpression is a mechanism of trastuzumab resistance in HER2+ breast cancer patients. Proc Natl Acad Sci U S A. 2011;108(9):3761-3766.

16. Zhang S, et al. Combating trastuzumab resistance by targeting SRC, a common node downstream of multiple resistance pathways. Nat Med. 2011;17(4):461-469.

17. Dua R, Zhang J, Nhonthachit P, Penuel E, Petropoulos C, Parry G. EGFR over-expression and activation in high HER2, ER negative breast cancer cell line induces trastuzumab resistance. Breast Cancer Res Treat. 2010;122(3):685-697.

18. Gallardo A, et al. Increased signalling of EGFR and IGF1R, and deregulation of PTEN/PI3K/ Akt pathway are related with trastuzumab resistance in HER2 breast carcinomas. Br J Cancer. 2012;106(8):1367-1373.

19. Ghosh R, et al. Trastuzumab has preferential activity against breast cancers driven by HER2 homodimers. Cancer Res. 2011;71(5):1871-1882.

20. Lee-Hoeflich ST, et al. A central role for HER3 in HER2-amplified breast cancer: implications for targeted therapy. Cancer Res. 2008;68(14):5878-5887.

21. Aurisicchio L, et al. Novel anti-ErbB3 monoclonal antibodies show therapeutic efficacy in xenografted and spontaneous mouse tumors. J Cell Physiol. 2012;227(10):3381-3388.

22. Foreman PK, et al. ErbB3 inhibitory surrobodies inhibit tumor cell proliferation in vitro and in vivo. Mol Cancer Ther. 2012;11(7):1411-1420.

23. Hickinson DM, et al. AZD8931, an equipotent, reversible inhibitor of signaling by epidermal growth factor receptor, ERBB2 (HER2), and ERBB3: a unique agent for simultaneous ERBB receptor blockade in cancer. Clin Cancer Res. 2010;16(4):1159-1169.

24. McDonagh CF, et al. Antitumor activity of a novel bispecific antibody that targets the ErbB2/ ErbB3 oncogenic unit and inhibits heregulininduced activation of ErbB3. Mol Cancer Ther. 2012;11(3):582-593.

25. Hong J, Katsha A, Lu P, Shyr Y, Belkhiri A El-Rifai W. Regulation of ERBB2 receptor by t-DARPP mediates trastuzumab resistance in human esophageal adenocarcinoma. Cancer Res. 2012;72(17):4504-4514

26. Dulak AM, et al. Gastrointestinal adenocarcinomas of the esophagus, stomach, and colon exhibit distinct patterns of genome instability and oncogenesis. Cancer Res. 2012;72(17):4383-4393.

27. Dulak AM, et al. Exome and whole-genome sequencing of esophageal adenocarcinoma identifies recurrent driver events and mutational complexity. Nat Genet. 2013;45(5):478-486.

28. Beroukhim R, et al. The landscape of somatic copy-number alteration across human cancers. Nature. 2010;463(7283):899-905.

29. Deng N, et al. A comprehensive survey of genomic alterations in gastric cancer reveals systematic patterns of molecular exclusivity and co-occurrence among distinct therapeutic targets. Gut. 2012;61(5):673-684.

30. Cancer Genome Atlas Network. Comprehensive molecular characterization of gastric adenocarcinoma. Nature. 2014;513(7517):202-209.

31. Karst AM, et al. Cyclin E1 deregulation occurs early in secretory cell transformation to promote formation of fallopian tube-derived high-grade serous ovarian cancers. Cancer Res. 2014;74(4):1141-1152.

32. Cancer Genome Atlas Network. Comprehensive molecular portraits of human breast tumours. Nature. 2012;490(7418):61-70.

33. Cheung LW, et al. High frequency of PIK3R1 and PIK3R2 mutations in endometrial cancer elucidates a novel mechanism for regulation of PTEN protein stability. Cancer Discov. 2011;1(2):170-185.

34. Barretina J, et al. The Cancer Cell Line Encyclopedia enables predictive modelling of anticancer drug sensitivity. Nature. 2012;483(7391):603-607.

35. Okamoto W, et al. Antitumor action of the MET tyrosine kinase inhibitor crizotinib (PF-02341066) in gastric cancer positive for MET amplification. Mol Cancer Ther. 2012;11(7):1557-1564.

36. Kuhn E, et al. Identification of molecular pathway aberrations in uterine serous carcinoma by genome-wide analyses. J Natl Cancer Inst. 2012;104(19):1503-1513.

37. Natrajan R, et al. Functional characterization of the 19q12 amplicon in grade III breast cancers. Breast Cancer Res. 2012;14(2):R53.

38. Zhao S, et al. Landscape of somatic singlenucleotide and copy-number mutations in uterine serous carcinoma. Proc Natl Acad Sci US A. 2013;110(8):2916-2921.

39. Jiang $\mathrm{H}$, Edgar BA. EGFR signaling regulates the proliferation of Drosophila adult midgut progenitors. Development. 2009;136(3):483-493.

40. Jiang H, Grenley MO, Bravo MJ, Blumhagen RZ, Edgar BA. EGFR/Ras/MAPK signaling mediates adult midgut epithelial homeostasis and regeneration in Drosophila. Cell Stem Cell. 2011;8(1):84-95.

41. Ritter CA, et al. Human breast cancer cells selected for resistance to trastuzumab in vivo overexpress epidermal growth factor receptor and ErbB ligands and remain dependent on 
the ErbB receptor network. Clin Cancer Res. 2007;13(16):4909-4919.

42. Janjigian $Y Y$, et al. Monitoring afatinib treatment in HER2-positive gastric cancer with 18FFDG and 89Zr-trastuzumab PET. J Nucl Med. 2013;54(6):936-943.

43. Schaefer G, Shao L, Totpal K, Akita RW. Erlotinib directly inhibits HER2 kinase activation and downstream signaling events in intact cells lacking epidermal growth factor receptor expression. Cancer Res. 2007;67(3):1228-1238.

44. Wang L, et al. PI3K pathway activation results in low efficacy of both trastuzumab and lapatinib. BMC Cancer. 2011;11:248.

45. Chen CT, Kim H, Liska D, Gao S, Christensen JG, Weiser MR. MET activation mediates resistance to lapatinib inhibition of HER2-amplified gastric cancer cells. Mol Cancer Ther. 2012;11(3):660-669.

46. Waddell T, et al. Epirubicin, oxaliplatin, and capecitabine with or without panitumumab for patients with previously untreated advanced oesophagogastric cancer (REAL3): a randomised, open-label phase 3 trial. Lancet Oncol. 2013;14(6):481-489.

47. Lordick F, et al. Capecitabine and cisplatin with or without cetuximab for patients with previously untreated advanced gastric cancer (EXPAND): a randomised, open-label phase 3 trial. Lancet Oncol. 2013;14(6):490-499.

48. Kwak EL, et al. Phase 2 trial of afatinib, an ErbB family blocker, in solid tumors genetically screened for target activation. Cancer. 2013;119(16):3043-3051.

49. Bang YJ, et al. A randomized, open-label, phase III study of lapatinib in combination with weekly paclitaxel versus weekly paclitaxel alone in the second-line treatment of HER2 amplified advanced gastric cancer (AGC) in Asian population: Tytan study. Gastrointestinal Cancers Symposium. 2013; abstract 11.

50. Lee HE, et al. Clinical significance of intratumoral HER2 heterogeneity in gastric cancer. Eur
J Cancer. 2013;49(6):1448-1457.

51. Mermel CH, Schumacher SE, Hill B, Meyerson ML, Beroukhim R, Getz G. GISTIC2.0 facilitates sensitive and confident localization of the targets of focal somatic copy-number alteration in human cancers. Genome Biol. 2011;12(4):R41.

52. Wagle N, et al. High-throughput detection of actionable genomic alterations in clinical tumor samples by targeted, massively parallel sequenc ing. Cancer Discov. 2012;2(1):82-93.

53. Chou TC. Drug combination studies and their synergy quantification using the Chou-Talalay method. Cancer Res. 2010;70(2):440-446.

54. Penuel E, Akita RW, Sliwkowski MX. Identification of a region within the ErbB2/HER2 intracellular domain that is necessary for ligand-independent association. J Biol Chem. 2002;277(32):28468-28473.

55. Hu Y, et al. HER2 amplification, overexpression and score criteria in esophageal adenocarcinoma. Mod Pathol. 2011;24(7):899-907. 